\title{
Characterization studies of Silicon Photomultipliers and crystals matrices for a novel time of flight PET detector
}

\author{
Etiennette Auffray ${ }^{3}$, Faraah Ben Mimoun Bel Hadj ${ }^{3}$, Daniele Cortinovis ${ }^{* 1,2}$, Katayoun Doroud $^{3}$, Erika Garutti ${ }^{2}$, Paul Lecoq ${ }^{3}$, \\ Zheng $\mathrm{Liu}^{4}$, Rosana Martinez ${ }^{4}$, Marco Paganoni ${ }^{4}$, Marco Pizzichemi ${ }^{4}$, Alessandro Silenzi ${ }^{1}$, Chen Xu ${ }^{1,2}$ and Milan Zvolský ${ }^{1,2}$ \\ ${ }^{1}$ Deutches Elektronen-Synchrotron (DESY), Notkestrasse 85, 22607 Hamburg, Germany \\ ${ }^{2}$ University of Hamburg, Luruper Chaussee 149, 22671 Hamburg, Germany \\ ${ }^{3}$ European Organization for Nuclear Research (CERN), 1211, Geneva 23, Switzerland \\ ${ }^{4}$ University of Milano-Bicocca, Piazza della Scienza 3, 20126 Milano, Italy
}

\begin{abstract}
This paper describes the characterization of crystal matrices and silicon photomultiplier arrays for a novel Positron Emission Tomography (PET) detector, namely the external plate of the EndoTOFPET-US system. The EndoTOFPET-US collaboration aims to integrate Time-Of-Flight PET with ultrasound endoscopy in a novel multimodal device, capable to support the development of new biomarkers for prostate and pancreatic tumors. The detector consists in two parts: a PET head mounted on an ultrasound probe and an external PET plate. The challenging goal of $1 \mathrm{~mm}$ spatial resolution for the PET image requires a detector with small crystal size, and therefore high channel density: 4096 LYSO crystals individually readout by Silicon Photomultipliers (SiPM) make up the external plate. The quality and properties of these components must be assessed before the assembly. The dark count rate, gain, breakdown voltage and correlated noise of the SiPMs are measured, while the LYSO crystals are evaluated in terms of light yield and energy resolution. In order to effectively reduce the noise in the PET image, high time resolution for the gamma detection is mandatory. The Coincidence Time Resolution (CTR) of all the SiPMs assembled with crystals is measured, and results show a value close to the demanding goal of 200 ps FWHM. The light output is evaluated for every channel for a preliminary detector calibration, showing an average of about 1800 pixels fired on the SiPM for a $511 \mathrm{keV}$ interaction. Finally, the average energy resolution at $511 \mathrm{keV}$ is about $13 \%$, enough for effective Compton rejection.
\end{abstract}

\section{Introduction}

Positron Emission Tomography (PET) is a non invasive, diagnostic imaging technique for measuring the metabolic activity of cells in the human body. Nowadays it is widely used for cancer diagnosis [1].

Among the tumors, pancreatic carcinoma is one of the most aggressive and resistant to current therapies [2], and most often is detected only on an advanced state of development. On the other hand, prostate cancer is the most common cancer among males [3].

Both pancreas and prostate are surrounded by organs with high uptake: the liver and the heart are close to the pancreas while the bladder is near the prostate. In these cases the current full-body multimodal PET scanners have strong limitations, due to the noise from the neighboring organs and the lack of specific biomarkers [4] [5]. EndoTOFPET-US [6] aims to overcome these limits, allowing to investigate the performances of newly developed specific biomarkers of tumoral processes for pancreas and prostate. Both organs are usually exam- ined with ultrasound probes through natural cavities, therefore the diagnostic capability can be enhanced by fusing the morphological image from ultrasound with a PET metabolic image. The endoscopic approach and the use of the TOF information with an unprecedented CTR of 200 ps ( $3 \mathrm{~cm}$ along the line of response) allow to define a specific Region Of Interest (ROI) and significantly suppress the background from the neighboring organs.

The EndoTOFPET-US detector consists of a PET head extension mounted on a commercial US endoscope placed close to the ROI and an external PET plate facing the patient's abdomen, in coincide with the PET head (Figure 11. The external plate is a square of $23 \times 23 \mathrm{~cm}^{2}$, and it is made of 256 detector unit modules. Each module consists in a $4 \times 4$ LYSO:Ce crystal matrix glued to a discrete array of $4 \times 4$ analog SiPMs from Hamamatsu. LYSO:Ce crystals have high light yield and fast decay time, therefore appropriate for TOF PET. SiPMs are novel photon detectors also suitable for TOF PET applications [7], thanks to their compactness, high gain,

\footnotetext{
*Corresponding author, daniele.cortinovis@desy.de
} 
insensitivity to magnetic fields, low operating voltage and excellent timing properties.

The following sections describe the experimental setups and the results of the characterization of the SiPMs, crystals and the combined system (SiPM + crystal). All the setups have been designed for a fast and reliable measurement of a large quantity of components, rather than a detailed study of single devices.

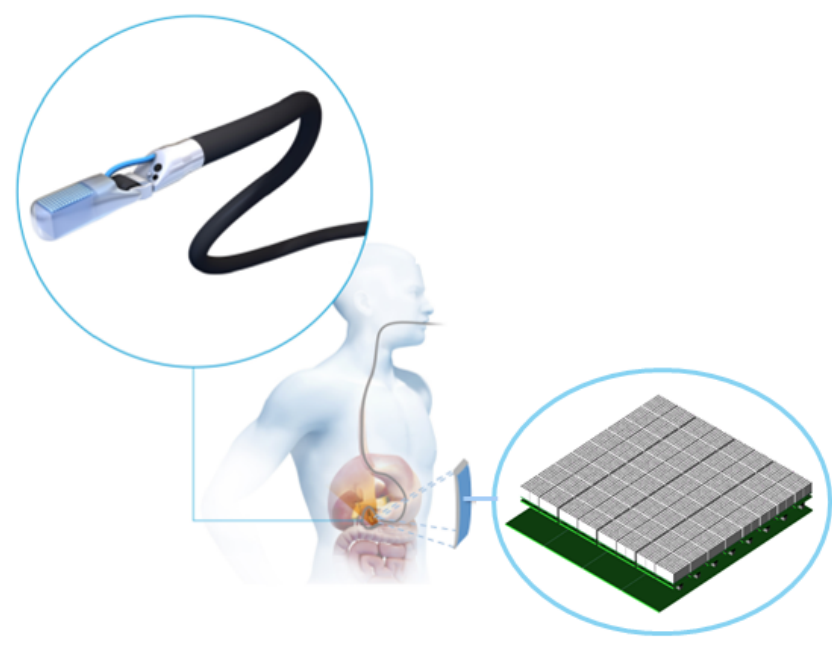

Figure 1: The EndoTOFPET-US detector with a magnification of the pancreatic endoscope (top circle) and the external plate (bottom circle).

\section{Photodetector Characterization}

The SiPMs chosen for the external plate are matrices of $4 \times 4$ discrete MPPCs (Multi Pixel Photon Counter) from Hamamatsu photonics (S12643-050CN). Each SiPM in the matrix is a square of $3.0 \times 3.0 \mathrm{~mm}^{2}$ with 3464 active pixels (50 x $50 \mu \mathrm{m}^{2}$ each). The distance between two adjacent SIPMs in the matrix (center to center) is $3.6 \mathrm{~mm}$. These SiPMs exploit the Through Silicon Via (TSV) technology, leading to less dead space and a reduced connection length as compared to conventional wire-bonded SiPM. The following sections describe the experimental setup, the analysis method and the results of the characterization of $256 \mathrm{SiPM}$ matrices.

\subsection{Experimental setup}

The layout of the experimental laboratory setup is shown in Figure 2. In a light tight box, the SiPM matrix is mounted on a motherboard incorporating linear amplifiers (Infineon BGA614) and high voltage filters. The signal for each of the $16 \mathrm{SiPMs}$ of the matrix is obtained using low intensity blue light (Advanced laser diode system, $451 \mathrm{~nm}$ ). The amplified SiPM output is readout by a VME-based charge-to-digital converter (CAEN QDC965A) with a resolution of $25 \mathrm{fC}$ per QDC bin. The heat generated by the active components on the SiPM motherboard is dissipated by a fan. The temperature is monitored by a sensor (Dallas DS18B20) but not actively controlled.

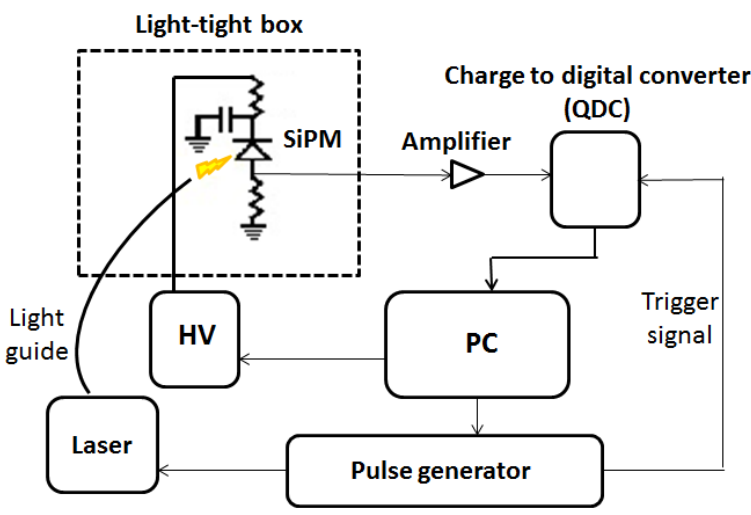

Figure 2: Sketch of the experimental setup for the SiPM characterization.

\subsection{Gain and Breakdown voltage}

The integrated charge spectrum given by the light pulses in shown in Figure 3 . This is repeated for 30 voltage steps, acquiring 200k events sample per step with an integration gate of $100 \mathrm{~ns}$ synchronized with the laser pulse. The first peak corresponds to the pedestal (zero pixel fired) while the following peaks correspond to an increasing number of pixels fired. After the pedestal subtraction, the spectrum is projected into the frequency space using a Fast Fourier Transform. The period of the first harmonic is taken as the value of the SiPM gain $(G)$ in QDC units, which is then converted into number of electrons by the following formula:

$$
G=\frac{G \cdot R_{q d c}}{A}
$$

where $R_{q d c}$ is the QDC resolution of $25 \mathrm{fC}$ per QDC bin and $A$ is the amplification factor of the readout board (19 $\mathrm{dB}, \sim 8.9)$. The error on the gain is about $1 \%$, obtained as one bin in the Fourier space plus an additional uncertainty of $5 \%$, evaluated by repeating the measurement multiple times under the same conditions. 


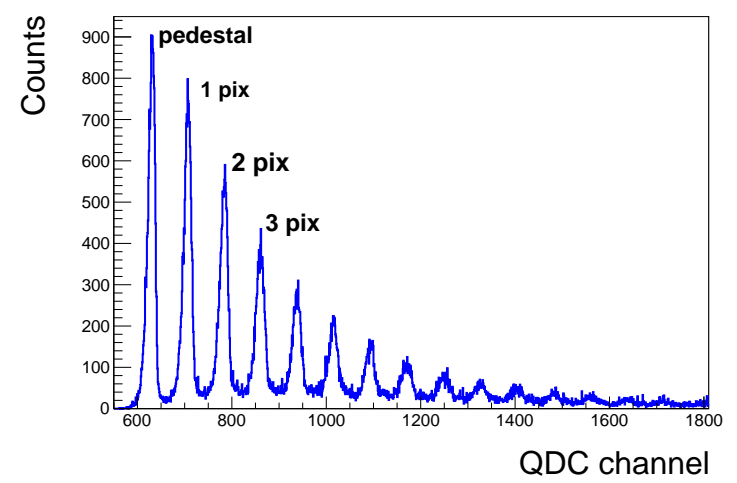

Figure 3: Single photo-electron spectrum for a single SiPM, each peak corresponds to a certain number of pixel fired.

Figure 4 shows the gain dependence on the voltage bias applied. As expected, the gain is linearly proportional to the bias voltage applied, according to

$$
G=C_{\text {pixel }} \cdot \Delta U=C_{\text {pixel }}\left(U_{\text {bias }}-U_{b d}\right),
$$

where $C_{\text {pixel }}$ is the pixel capacitance, $\Delta U$ is the excess bias voltage, $U_{b i a s}$ is the applied bias voltage and $U_{b d}$ is the breakdown voltage. Hence $U_{b d}$ is obtained by applying a linear fit and extrapolating to zero gain, with an uncertainty of the order of a few tens on $\mathrm{mV}$. Figure 5 shows the distribution of the gain slope ( $G$ at $1 \mathrm{~V}$ excess bias) for all the SiPMs. The mean value is $0.48 \cdot 10^{6}$ $\mathrm{V}^{-1}$ with a spread of $3 \%$ among the SiPMs tested.

It is known that the $U_{b d}$ depends on the temperature [8], and some fluctuations have occurred during the whole set of measurements. Moreover, local differences in temperature have been observed within the SiPM matrix, due to the inhomogeneous dissipation of the heat generated by the amplifiers. Therefore an offline temperature correction (see section 2.5) has been applied in order to compare the results. Figure 6 shows the distribution of the breakdown voltages at $25^{\circ} \mathrm{C}$ for all the SiPMs.

Although the $U_{b d}$ spread for all the SiPM is about $2 \mathrm{~V}$, this must not exceed $0.5 \mathrm{~V}$ in each single SiPM matrix, because this is the maximum voltage bias tuning range of the ASIC developed for the SiPM readout [9] [10]. As shown in Figure 7, all the SiPM matrices respect this requirement.

Finally, the results obtained have been compared to the operating voltages $\left(U_{o p}\right)$ provided by the SiPM producer. The $U_{o p}$ is defined as: $G\left(U_{O P}\right)=1.25 \cdot 10^{6}$. The differences between the $U_{o p}$ calculated using equation 2 with data from the measurements and $U_{o p}$ provided by Hamamatsu are shown in Figure 8 . The agreement is good, despite a shift of $6 \%$ and a spread of $10 \%$. This is due to the different measurement method implied by the SiPM producer and the uncertainty of $U_{b d}$ and $G$.

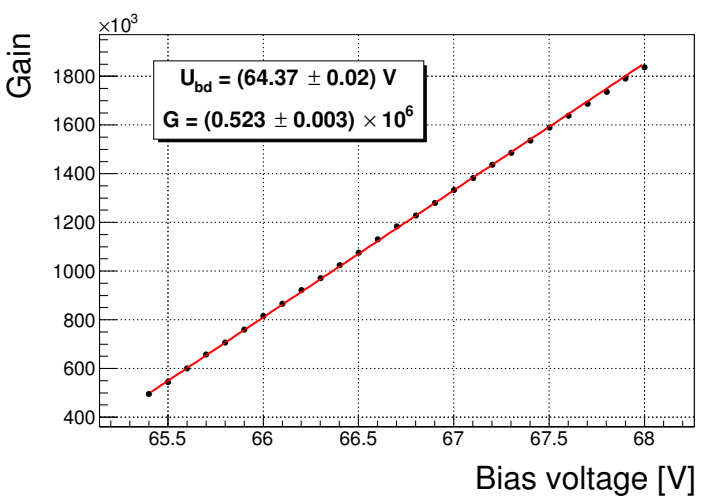

Figure 4: Gain as a function of the applied bias voltage for a single SiPM. The red line is a linear fit on the data points whose errors are within the dots. The breakdown voltage $\left(U_{b d}\right)$ and gain $(G)$ at $1 \mathrm{~V}$ excess bias obtained from the fit are given in the inlet.

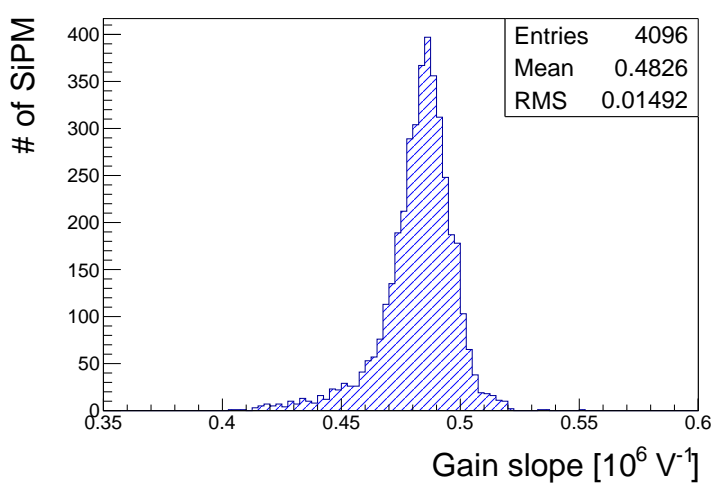

Figure 5: Distribution of the gain slope (gain per volt) for all the SiPMs.

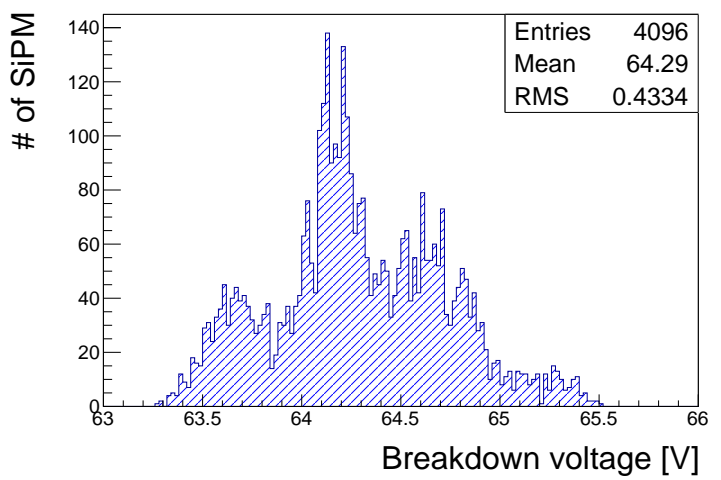

Figure 6: Distribution of the extracted breakdown voltage for all the SiPMs. 


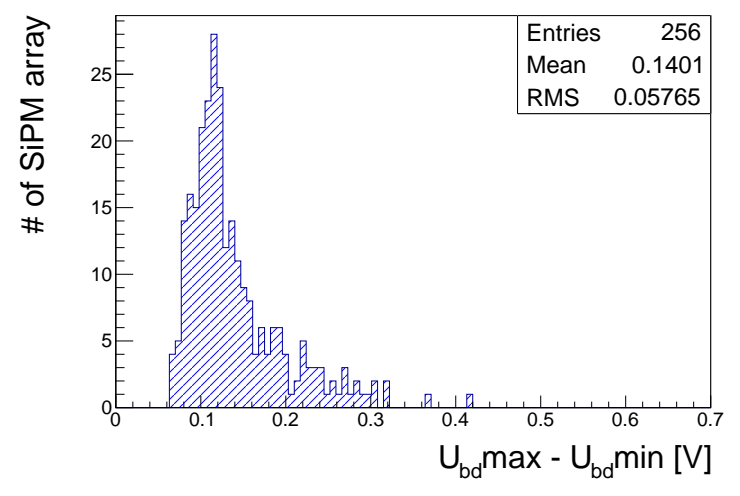

Figure 7: Distribution of the maximum $\Delta U_{b d}$, i.e. the difference between maximum and minimum $U_{b d}$ in one matrix. The deviation is below the $0.5 \mathrm{~V}$ requirement specified to the SiPM producer.

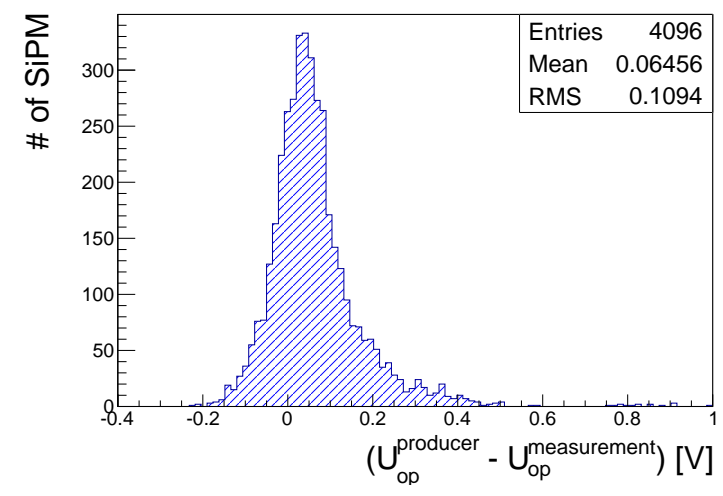

Figure 8: Difference between $U_{o p}$ given by the SiPM producer and $U_{\text {op }}$ calculated from the measurement.

\subsection{Dark Count Rate}

Even in absence of light, an avalanche breakdown can be triggered by a photoelectron generated from thermal excitation or by tunneling effect. This phenomenon, usually called Dark Count Rate (DCR), is Poisson distributed and it is measured by randomly integrating the charge with a time window $\Delta t$ of 100 ns (same time window as for the gain measurement). A typical noise spectrum is visible in Figure 9

The probability of having two dark events in the time $\Delta t$ is very low, therefore the events corresponding to more than 1 pixel fired are mainly due to correlated noise, which will be described in the next section.

The events in the pedestal peak, i.e. the events with charge less than 0.5 pixel threshold, are not affected by correlated noise, hence they are used to calculate the uncorrelated DCR, defined as $D C R_{0.5 p i x}$.

In a time range $\Delta t$, the expected number of $D C R_{0.5 p i x}$ events $n$ is given by $n=D C R_{0.5 p i x} \cdot \Delta t$. The number of events in the pedestal distribution is given by the Poisson probability density function for zero $D C R_{0.5 p i x}$ events in the time $\Delta t$ :

$$
P_{0}(\Delta t)=e^{-D C R_{0.5 p i x} \cdot \Delta t}
$$

The $D C R_{0.5 p i x}$ is therefore calculated using:

$$
D C R_{0.5 p i x}=\frac{\ln \left(N_{0} / N_{\text {total }}\right)}{\Delta t},
$$

where $N_{0}$ is the number of events in the pedestal peak and $N_{\text {total }}$ is the total number of events recorded. The error on the calculated value depends on the statistic of the sample and on the quality of the spectrum.

For each SiPM the $D C R_{0.5 \text { pix }}$ is evaluated for 30 voltage levels, in a range of $3 \mathrm{~V}$ starting about $1 \mathrm{~V}$ above the breakdown voltage. 500k event samples are collected for each voltage point.

Also the DCR has a dependence on the temperature, therefore a correction (see section 2.5 has been applied in order to compensate for temperature variations.

The distribution of $D C R_{0.5 p i x}$, corrected to $25^{\circ} \mathrm{C}$ and at $2.5 \mathrm{~V}$ excess bias, is shown in Figure 10 for all the SiPMs.

The DCR has a negative impact on the time resolution. The best time resolution is achieved when the timing threshold on the readout ASIC is set as low as possible, up to the noise level. Therefore a $D C R_{0.5 \text { pix }}$ acceptance limit of $3 \mathrm{MHz}$ for each SiPM has been agreed with the SiPM producer. Almost all the SiPMs are within the limit, however 5 matrices have been sent back to the producer because they included at least one SiPM with excessive $D C R_{0.5 \text { pix }}$.

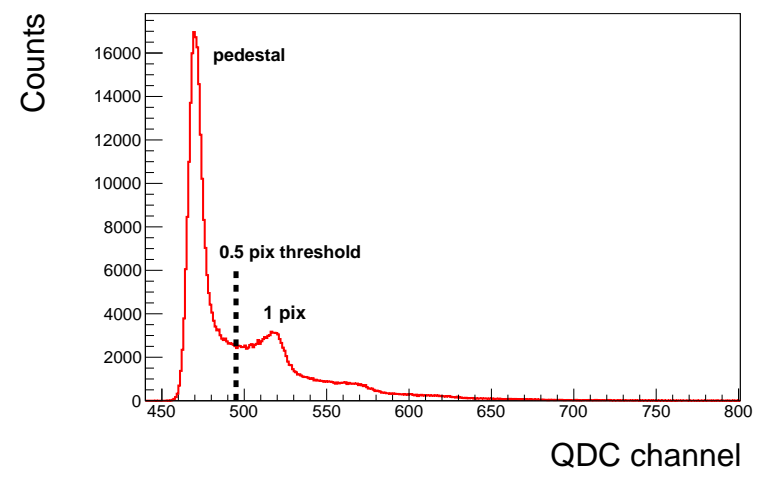

Figure 9: DCR charge spectrum. The events below the 0.5 pix threshold (pedestal peak) are used to calculate $D C R_{0.5 \text { pix }}$. 


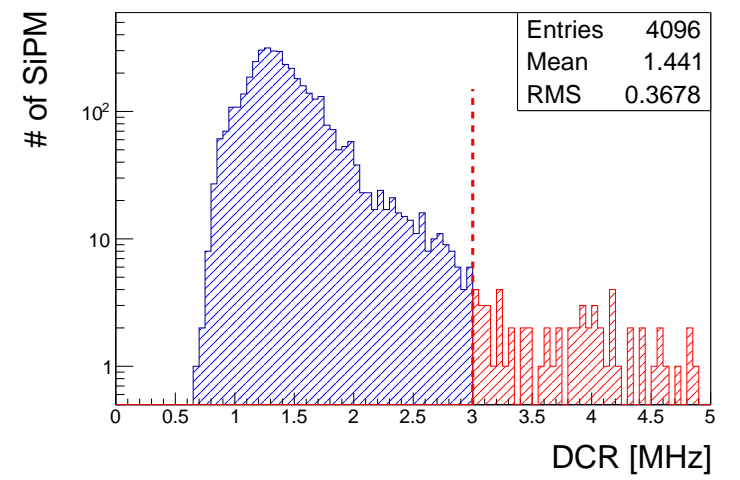

Figure 10: Distribution of the $D C R_{0.5 p i x}$ at $2.5 \mathrm{~V}$ excess bias and at $25^{\circ} \mathrm{C}$ for all the SiPMs. The red line indicates the threshold of acceptable DCR. Entries marked in red correspond to the rejected SiPMs.

\subsection{Correlated noise}

Correlated noise includes after-pulse and inter-pixel optical crosstalk [11].

After-pulses are generated if an electron produced during an avalanche is trapped and released at a later time, with a delay ranging from nanoseconds up to several microseconds. Depending on the trapping time constant and the pixel recovery time, trapped electrons can generate an avalanche of equivalent or smaller charge with respect to the DCR pulses.

Inter-pixel crosstalk is due to optical photons generated during a pixel breakdown. These photons have a certain probability to reach the neighboring pixels, triggering new avalanches.

The precise measurement of each of the two effects is beyond the scope of this study. However, it's possible to estimate the correlated noise triggering probability $P_{c n}$ as:

$$
P_{c n}=\frac{D C R_{1.5 p i x}}{D C R_{0.5 p i x}}
$$

where $D C R_{1.5 p i x}$ is calculated by setting the threshold to 1.5 pixel fired. Figure 11 shows the correlated noise probability at $2.5 \mathrm{~V}$ excess bias for all the SiPMs. The average results obtained, about $30 \%$, is compatible with measurements on similar devices and does not represent an issue for the detector operation.

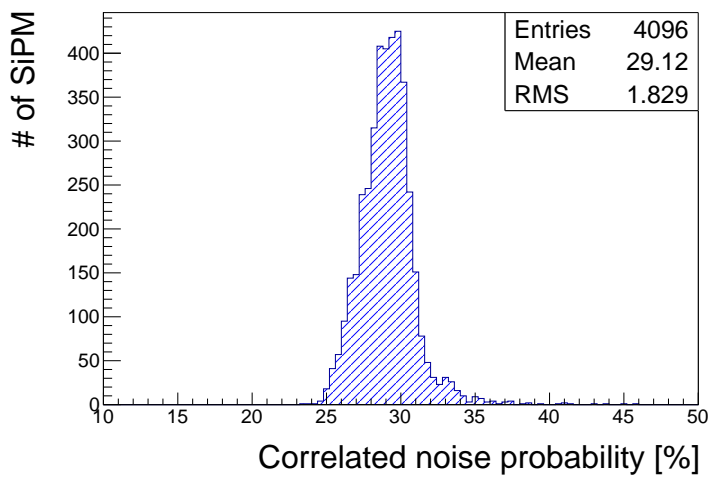

Figure 11: Correlated noise probability $P_{c n}$ at $2.5 \mathrm{~V}$ excess bias.

\subsection{Temperature dependence}

As mentioned in previous sections, SiPM properties depend on temperature. Therefore, DCR, gain, breakdown voltage and correlated noise have been measured at different temperatures for a single SiPM in an array. Using a climate chamber (Espec LU-123), several voltage scans have been performed in a temperature range between $6{ }^{\circ} \mathrm{C}$ and $30{ }^{\circ} \mathrm{C}$. Figure 12 shows the linear temperature dependence of the breakdown voltage; the uncertainty on the temperature is included but it is smaller than the marker size. The coefficient obtained from the linear fit is $70.1 \mathrm{mV} /{ }^{\circ} \mathrm{C}$, and is used for the temperature corrections applied in section 2.2 and 4.1 . The dependence of the DCR as a function of the temperature should scale with the density of the thermal carriers [12] according to equation:

$$
n(T)=C_{\text {pixel }} T^{3 / 2} e^{-\frac{E_{a}}{k_{B} T}},
$$

where $C_{\text {pixel }}$ is the pixel capacitance, $T$ is the temperature of the SiPM in Kelvin, $E_{a}$ is the activation energy and $k_{B}$ is the Boltzmann constant.

Figure 13 shows the DCR as a function of temperature at $2.5 \mathrm{~V}$ excess bias. An activation energy $E_{a}=0.6 \mathrm{eV}$ is obtained by fitting the data with equation 6 This value is compatible to previous studies [13], and it has been used for the temperature corrections applied in section 2.3.

The change in the gain is found to be less than $1 \% / 10^{\circ} \mathrm{C}$, which is negligible for the temperature variations occurred during the gain characterization of all the SiPMs. Also the correlated noise shows a mild dependence on the temperature, but it is not relevant for the detector performances. 


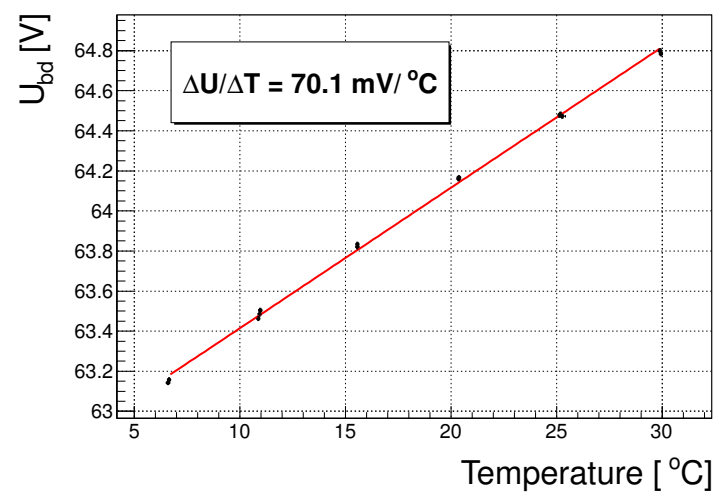

Figure 12: Breakdown voltage measured at different temperatures. The red curve is the linear fit to the data points. The coefficient obtained form the fit is displayed in the inlet.

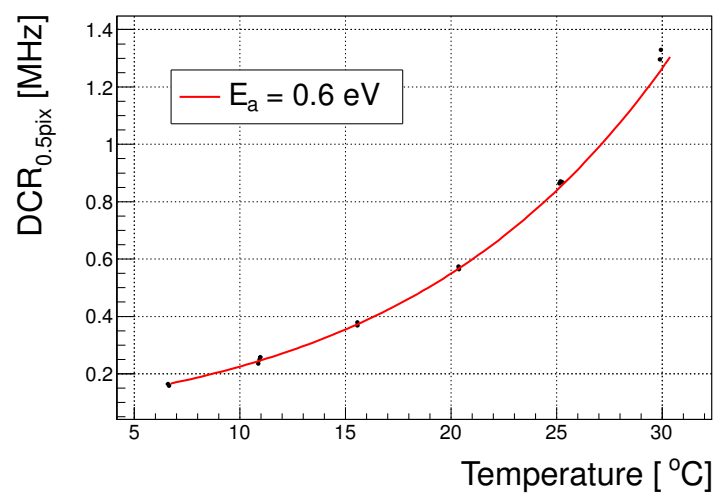

Figure 13: $D C R_{0.5 p i x}$ calculated at different temperatures. The red line is the fit to data points using equation 6 The value of $E_{a}$ obtained from the fit is displayed in the inlet.

\section{Crystals characterization}

The crystals for the external plate are grouped in 256 matrices of $4 \times 4$ LYSO:Ce scintillators, produced by Crystal Photonics Inc. The volume of each crystal is $3.5 \times 3.5 \times 15 \mathrm{~mm}^{3}$ and within the matrix they are separated by a reflector foil (ESR Vikuiti by $3 \mathrm{M}$ ). In order to ensure the system uniformity, measurements of light output and energy resolution have been performed on both faces for each matrix using the MiniACCOS setup [14], which allows a fast, automatic and highly reproducible data acquisition process. In this setup, each matrix is placed on a custom made teflon plate, for a total of 25 matrices per plate. An air gap of $5 \mathrm{~mm}$ is left between the matrix face and the PMT window. Systematic measurements were performed to evaluate uncertainties arising from the bench, yielding a total relative error of $4.7 \%$ for the light output and $12.8 \%$ for the energy resolution. Figure 14 shows the distribution of light yield obtained with MiniACCOS setup for all the 256 matrices. The distribution spread is $6.4 \%$, which subtracting the uncertainty of the setup gives a $4.3 \%$ as light output dispersion. Figure 15 shows the distribution of the energy resolution for all the modules, with a mean value of $13.4 \%$. The spread is comparable to the bench uncertainty, therefore the variations in energy resoluton are just due to the setup.

In the final configuration, each matrix will be glued to a SiPM array. The absolute values of light output obtained with the MiniACCOS setup have therefore to be rescaled to this configuration. In order to obtain this, light output for a subset of 13 matrices was measured on a standard XP2020Q PMT, using Rhodosil 47V grease as optical coupling and teflon as back wrapping. The scaling factor between this measurement condition and MiniACCOS was found to be 13.15, with a correlation coefficient of 0.89 between the two data sets, as shown in Figure 16

The average light yield for all the crystal matrices can therefore be derived as $32500 \pm 2700 \mathrm{Ph} / \mathrm{MeV}$, including in the evaluation of the uncertainty also the systematics arising from the calibration process. Based on the studies reported in [15], it can be estimated that at least $20000-25000 \mathrm{Ph} / \mathrm{MeV}$ are necessary to achieve a CTR of 200 ps FWHM, which make our set of matrices perfectly suitable for this purpose. The homogeneity on the crystals performance is also ensured within a $4.3 \%$ level for all 256 matrices tested with an energy resolution of $13.4 \pm 1.3 \%$ as mean value.

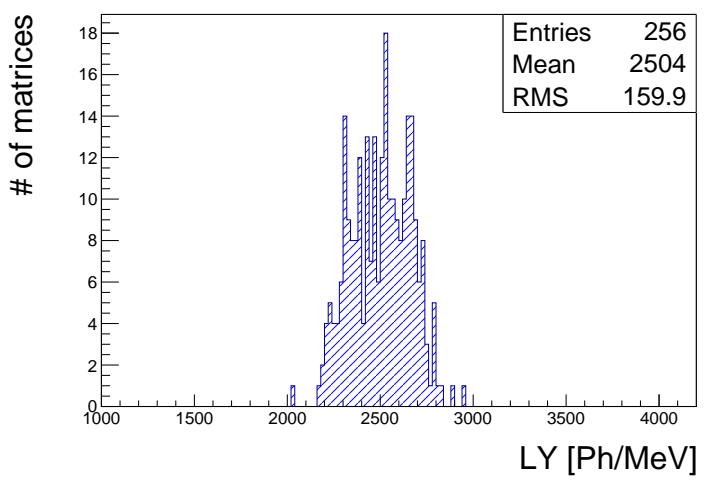

Figure 14: Light yield distributions for all the 256 crystal matrices. 


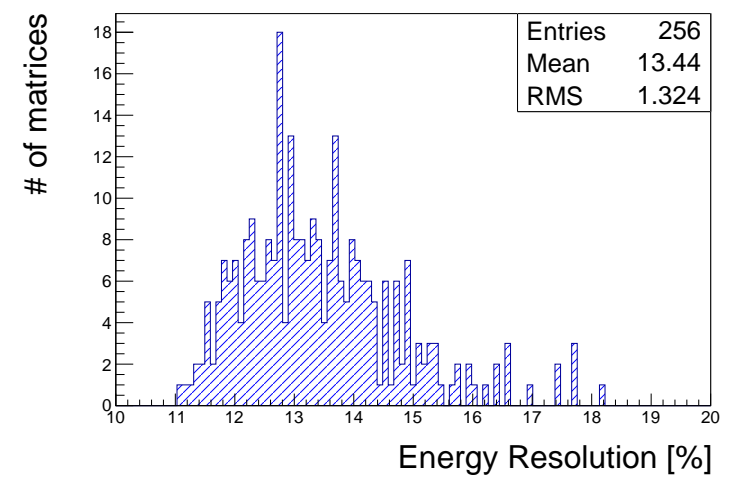

Figure 15: Energy resolution distributions for all the 256 crystal matrices.

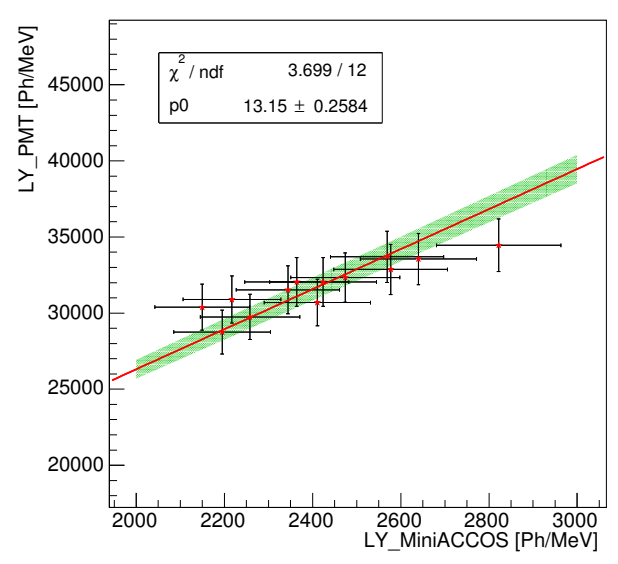

Figure 16: Correlated MiniACCOS-PMT data and the lineal fitting function with its $95 \%$ confidence interval band.

\section{Detector Module Characteriza- tion}

A dedicated bench has been developed to glue the crystal matrices on the SiPM arrays, allowing to assemble 5 modules per day. Figure 17 shows the setup: the crystal matrices are placed on a movable support and a camera allows the visual alignment with the SiPM matrices located under the crystals. The amount of glue (RTV3145) is calculated in order to have a maximum thickness of $0.1 \mathrm{~mm}$, and it is spread over the SiPM matrix with a dispenser. The SiPM matrix is then pushed againts the crystals and a visual inspection is performed to check if any air bubble occurred. If none, the modules are left on the support for the curing time ( $24 \mathrm{~h}$ ).

Every module is then measured regarding light output, energy resolution and CTR, which are described in the next sections.

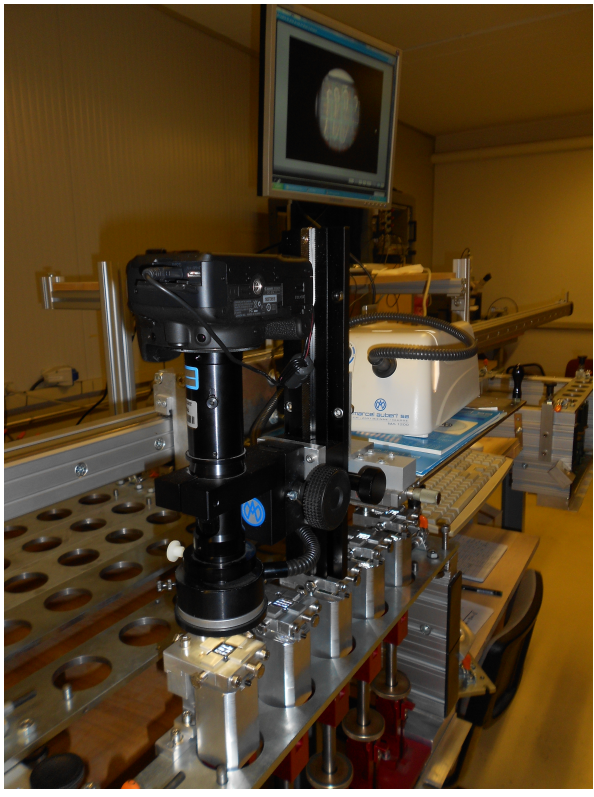

Figure 17: The bench for the module assembly. The binocular with the camera is placed over the 5 supports for the crystal matrices, the SiPM matrices (not visible) are located below.

\subsection{Detector light output}

The detector light output for the $511 \mathrm{keV}$ photon (defined here as $L O_{511}$ ) is of primary importance for the detector time resolution. As demonstrated in other studies [15], the number of detected photoelectrons must be maximized in order to achieve the best time resolution. Moreover, all the channels should have the same response for the $511 \mathrm{keV}$ photon, allowing an easier detector calibration.

$\mathrm{A}^{22} \mathrm{Na}$ source with an activity of about $1 \mathrm{MBq}$ is used as a $\beta^{+}$emitter. The setup is similar to the one described in section 2.1 but the SiPM signal is not amplified and the resolution of the QDC is set to $200 \mathrm{fC}$. The charge integration gate is set to $450 \mathrm{~ns}$ and it is triggered by the SiPM signal itself, using a discriminator (CAEN model 96) and a gate generator (LeCroy model 222). A channel switcher (Keithley 7002 switch system) provides automatic scan through the 16 channels in each matrix. The temperature is recorded before each channel measurement. Each SiPM is operated at $U_{o p}$ as defined in section 2.2. eventually corrected for temperature variations according to the coefficient of $70.1 \mathrm{mV} /{ }^{\circ} \mathrm{C}$ (see section 2.5.

Fig 18 shows a typical ${ }^{22} \mathrm{Na}$ spectrum for a single channel. The peak corresponding to the $511 \mathrm{keV}$ photon in the charge spectrum is fitted by a Gaussian function. Hence the light output $L O_{511}$, expressed in number of pixels fired on the SiPM, is obtained by the following relation:

$$
L O_{511}=\frac{(Q-P) \cdot r_{q d c}}{G},
$$


where $Q$ is the mean value of the Gaussian fit, $r_{q d c}$ is $200 \mathrm{fC}$ per bin, $P$ is the pedestal position, and $G$ is $1.25 \times 10^{6}$. The uncertainty on $L O_{511}$, of the order of about 30 pixels, is given by statistical error and temperature uncertainty of $0.5^{\circ} \mathrm{C}$. No error for the $U_{o p}$ is available.

The light output distribution for all the channels is shown in Figure 19, the average value of about 1800 pixels should allow to reach the CTR of 200 ps FWHM. A preliminary detector calibration will be based on these measurements.

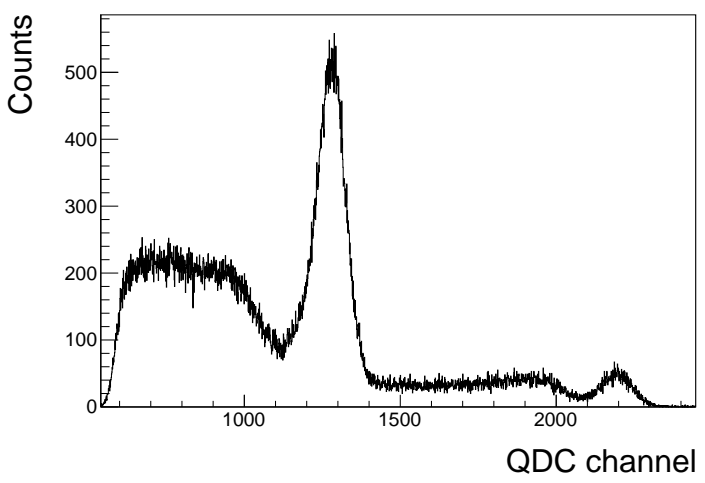

Figure 18: ${ }^{22} \mathrm{Na}$ spectrum for one channel. The higher peak corresponds to the full absorption of the $511 \mathrm{keV}$ photons, while the peak on the right comes from the $1277 \mathrm{keV}$ gamma emitted during the de-excitation of Neon to the ground state.

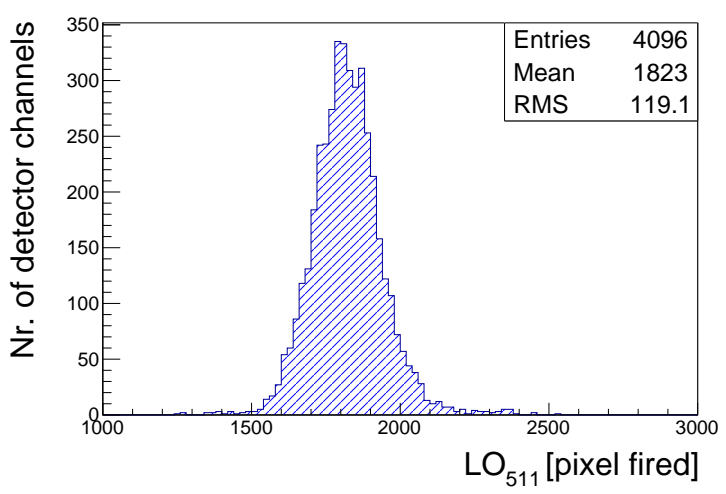

Figure 19: Light output distribution for all the channels.

\subsection{Inter channel cross-talk}

The cross-talk between the module channels is measured on a sample module. Similarly to the setup described in section 4.1. the ${ }^{22} \mathrm{Na}$ source is placed in front of the module and only one channel is chosen as trigger. Any other channel in the module is acquired simultaneously to the trigger, using a common integration gate of
450 ns in the QDC. The channel switcher provides the automatic scan trough the 15 channels, each operated at its own $U_{o p}$.

In every channel, only the events corresponding to the photopeak in the trigger are selected. Therefore, dividing the mean charge of these events by the photopeak position in the trigger, the cross-talk for the $511 \mathrm{keV}$ photon is obtained for all the remaining 15 channels in the module. The measurement is repeated by selecting different trigger channels in the module. Figure 20 shows the average crosstalk obtained from 4 different measurements: every time one of the central channels in the module is used as a trigger. The cross-talk does not exceed $20 \%$, and can be suppressed by setting a threshold at $100 \mathrm{keV}$.

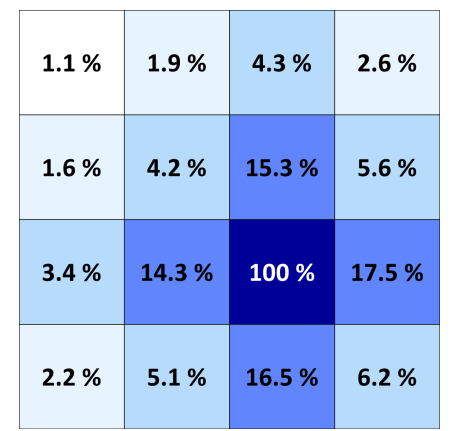

Figure 20: Average cross-talk for the $511 \mathrm{keV}$ photon obtained over 4 measurements, using each time a central channel as trigger.

\subsection{Energy calibration}

It is well known that the SiPM is a non-linear device due to the limited number of pixels, therefore a nonlinear correction is necessary in order to estimate the energy resolution for the $511 \mathrm{keV}$ gamma.

Using the setup described in section 4.1 the detector light output is measured for different gamma energies: $356 \mathrm{keV}$ from ${ }^{133} \mathrm{Ba}, 511$ and $1277 \mathrm{keV}$ from ${ }^{22} \mathrm{Na}$, and $662 \mathrm{keV}$ from ${ }^{137} \mathrm{Cs}$.

The measurement is performed on a sample of 4 modules (64 channels): for each channel the light output data are fitted with the function describing the SiPM saturation:

$$
N_{\text {pix }}=N_{\max }\left(1-e^{-\frac{\epsilon \cdot E}{N_{\max }}}\right),
$$

where $N_{\text {pix }}$ is the average number of pixels fired on the photodetector, $\epsilon$ is the number of pixel fired on the SiPM per unit of energy deposited in the crystal and $E$ is the energy of the detected gamma photon. $N_{\max }$ is related to the maximum number of pixels in the SiPM. This number is actually higher than the nominal value (3464 pixels) because the pixel recovery time is about $20 \mathrm{~ns}$, which is half of the decay constant of the crystal 
(40 ns).

Figure 21 shows the non-linear function for one channel, however this function is not unique for all the channels. It is expected that the parameter $\epsilon$ varies among the channels according to $L O_{511}$, which takes into account the variations in the SiPM properties (photo detection efficiency, correlated noise), crystal light yield and the optical coupling.

The dependence of $N_{\max }$ and $\epsilon$ to the $\mathrm{LO}_{511}$ for the 64 channels is shown in Figure 22 and 23 respectively. Although the linear dependence of $\epsilon$ was expected, also $N_{\max }$ shows a dependence on $L O_{511}$, maybe due to variations in in the pixel recovery time or crystal decay time. In both cases a linear fit is applied and the relations obtained are used to calculate $N_{\max }$ and $\epsilon$ for any other channel depending on its $L O_{511}$.

Figure 24 shows the energy obtained using equation 8 and the $L_{511}$ for every channel. Similarly, the corresponding energy of the $1277 \mathrm{keV}$ peak of the ${ }^{22} \mathrm{Na}$ spectrum is estimated for every channel (Figure 25). In both cases the predicted energy is in good agreement with the measurements, the precision worsens only if the channel $L O_{511}$ is significantly lower than the average.

Finally, after the energy calibration has been applied, the energy resolution for the $511 \mathrm{keV}$ gamma is evaluated for all the channels (Figure 26). Only one channel is out of the detector requirement: at least $20 \%$ is necessary to effectively discriminate the $511 \mathrm{keV}$ gammas from the Compton events.

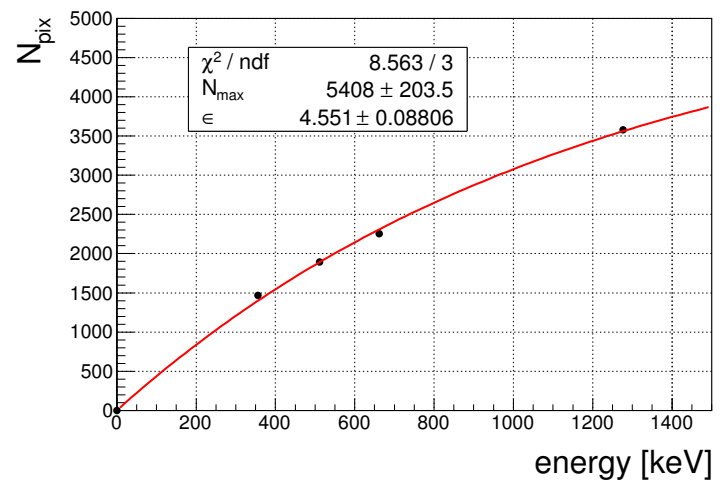

Figure 21: Detector light output for different gamma energies, the data are fitted with equation 8

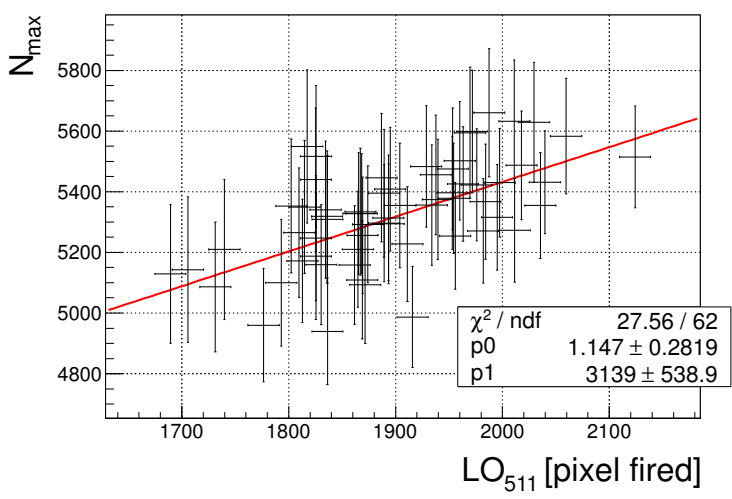

Figure 22: Distribution of the parameter $N_{\text {max }}$ of equation 8 depending on $\mathrm{LO}_{511}$ for 64 channels. The red line is the linear fit.

$\Psi$

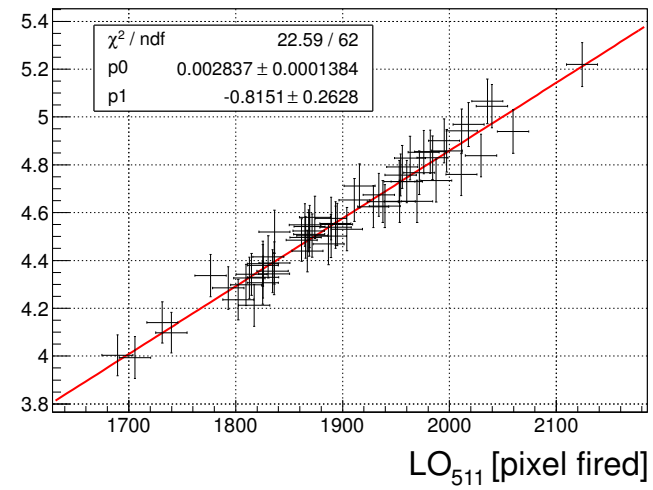

Figure 23: Distribution of the parameter $\epsilon$ of equation 8 depending on $\mathrm{LO}_{511}$ for 64 channels. The red line is the linear fit.

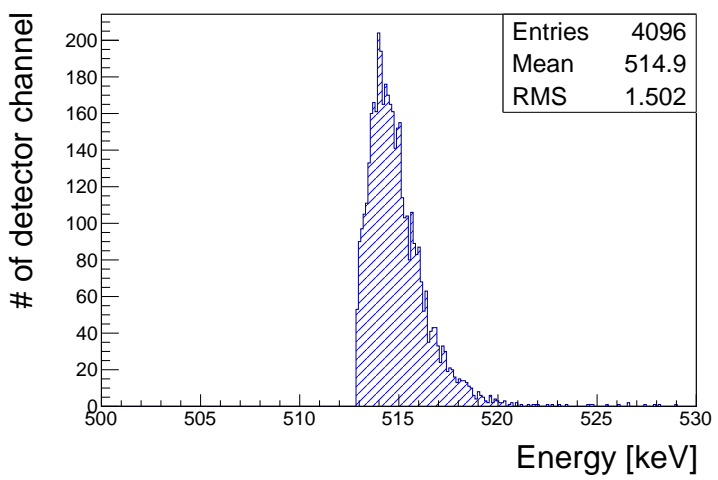

Figure 24: Energy Distribution calculated from the position of the $511 \mathrm{keV}$ peak (from ${ }^{22} \mathrm{Na}$ ) for all the channels after the energy correction has been applied. The tail on the right correspond to the channels with very low $\mathrm{LO}_{511}$. 


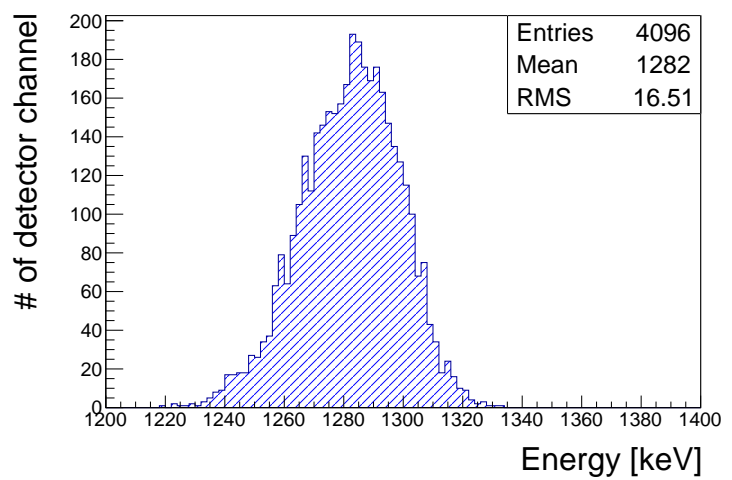

Figure 25: Energy Distribution calculated from the position of the $1277 \mathrm{keV}$ peak (from ${ }^{22} \mathrm{Na}$ ) for all the channels after the energy correction has been applied.

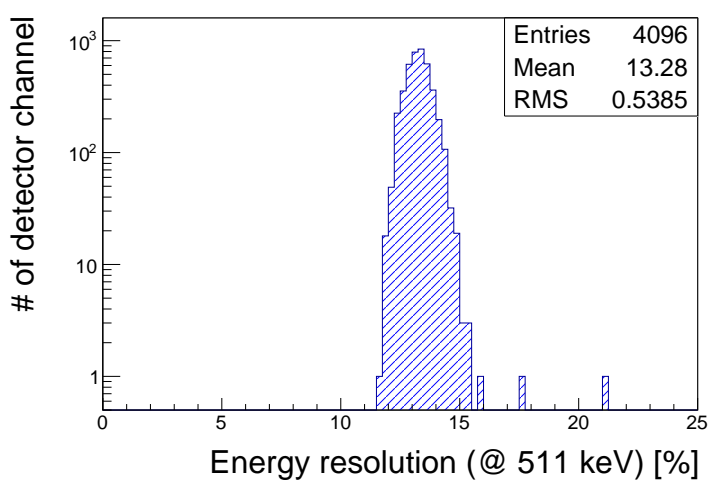

Figure 26: Energy resolution at $511 \mathrm{keV}$ for all the channels.

\subsection{Coincidence Time Resolution}

The coincidence time resolution of all the channels is measured using the setup shown in Figure 27. The two modules are facing each other at $10 \mathrm{~cm}$ distance, while a ${ }^{22} \mathrm{Na}$ point like source is placed between them on the line connecting the module centers. One module has been selected as the reference for the measurement of the other 256 modules.

The output signal of the SiPM is amplified and discriminated by NINO ASIC [16] and sent to a high precision TDC (HPTDC- 25ps LSB) [17]. The data analysis is performed with a total number of $4 \times 10^{6}$ triggers. As shown in Figure 28, the Time-over-Threshold (ToT) spectra are obtained for any two channels in coincidence; then a gaussian fit is applied to the peaks corresponding to the $511 \mathrm{keV}$ gamma events. Hence the time difference for the gamma events of the two channels are histogrammed and shown in Figure 29. A Gaussian fit is applied and the FWHM is taken as the CTR of the channel measured (227.3 ps $\pm 3.2 \mathrm{ps}$ in this case).
Figure 30 shows the CTR as a function of bias voltage for two channels in coincidence with the reference module. Due to the increase of the SiPM Photon Detection Efficiency (PDE) with the increase of excess bias, it is expected that also the CTR improves accordingly. However, also the DCR increase with the excess bias, and at a given point it becomes predominant. In order to compare the CTR of all the 256 modules, an excess bias of $2.5 \mathrm{~V}$ is used for any channel, the temperature is fixed at $19{ }^{\circ} \mathrm{C}$ and the NINO ASIC threshold is kept fixed at $55 \mathrm{mV}$, which is equivalent to the threshold of 0.5 photoelectron. The CTR distribution for all the channels, measured with respect to the reference module, is presented in Figure 31 and shows an average of $239 \pm$ 10 ps FWHM.

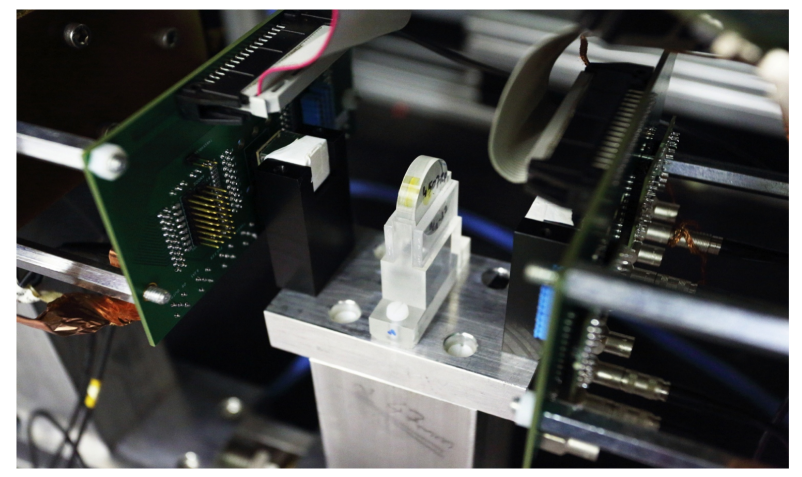

Figure 27: The setup used for the timing measurements. The two detector modules are placed facing each other, $10 \mathrm{~cm}$ apart. $A^{22} \mathrm{Na}$ source is placed between the two modules.
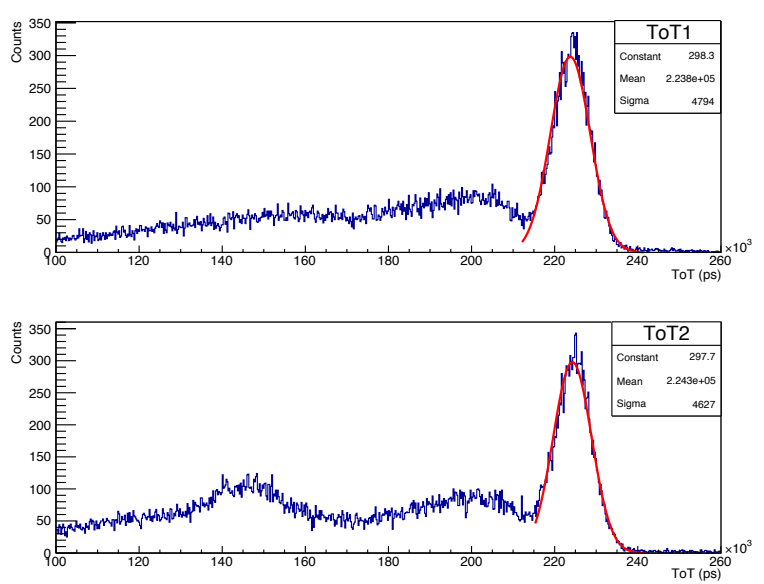

Figure 28: Typical Time over Threshold spectra for two channels in coincidence. 


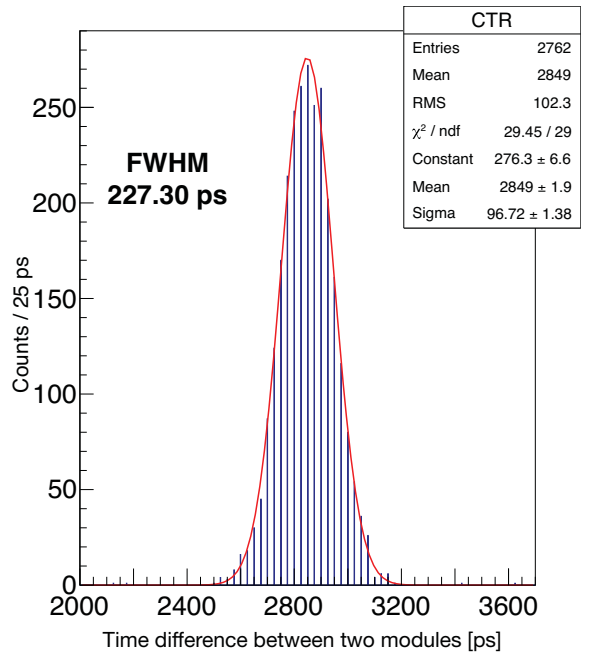

Figure 29: The CTR is the FWHM of a Gaussian fit of the time difference between each channel pair.

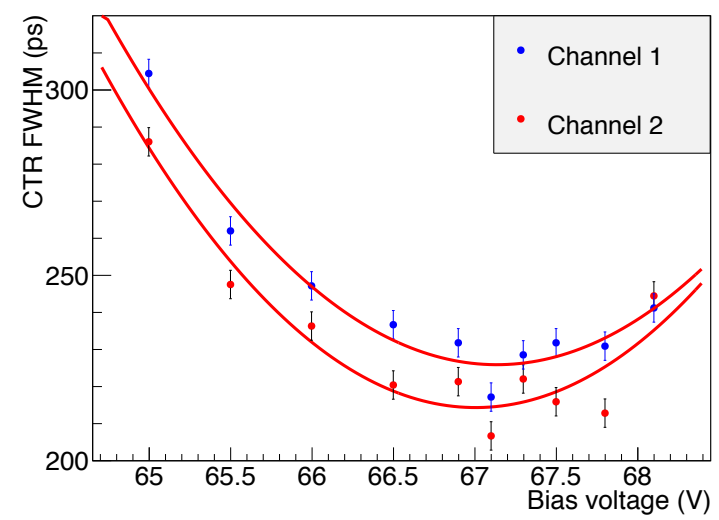

Figure 30: CTR as function of bias voltage for one pair of channels with respect to the reference module. Temperature fixed at $19^{\circ} \mathrm{C}$.

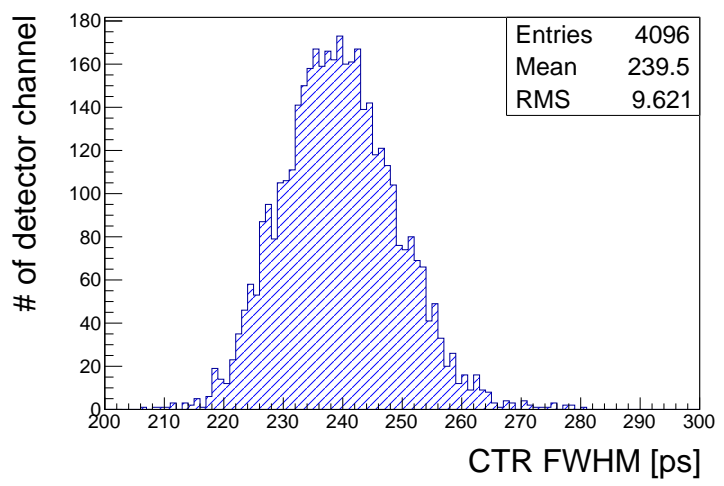

Figure 31: The CTR distribution of all channels pairs, at $19^{\circ} \mathrm{C}$ and at $2.5 \mathrm{~V}$ excess bias.

\section{Conclusions}

In the frame of the EndoTOFPET-US project, all the detector modules for the external plate have been characterized. The gain, breakdown voltage, DCR and correlated noise have been measured for all the received SiPMs, only $2 \%$ of them have been rejected due to excess DCR.

The LYSO:Ce crystal matrices show a very good light yield and a promising average CTR of $239 \pm 10$ ps FWHM has been obtained, which can be further improved in ideal conditions, such as lower temperature or optimal excess bias for each channel. The average energy resolution (at $511 \mathrm{keV}$ ) for all the modules is about $13 \%$, and it complies with the minimum requirement of $20 \%$. The light output for every channel is available for a preliminary detector calibration, and an average of about 1800 pixels fired for a $511 \mathrm{keV}$ gamma interaction has been obtained.

The detector modules are now ready for the integration with the dedicated ASICs and the final mechanical assembly.

\section{Acknowledgements}

The research leading to these results has received funding from the European Union Seventh Framework Programme [FP7/2007-2013] under Grant Agreement no. 256984, and is supported by a Marie Curie Early Initial Training Network Fellowship of the European Community's Seventh Framework Programme under contract number (PITN-GA-2011-289355-PicoSEC-MCNet).

\section{References}

[1] Bar-Shalom et al., Clinical performance of PET/CT in evaluation of cancer: additional value for diagnostic imaging and patient management, J. Nucl. Med., (2003) 44:1200-1209.

[2] P. Michl and T. Gress, Current Concepts and Novel Targets in Advanced Pancreatic Cancer, Gut. 2013;62(2):317-326.

[3] R. Siegel et al., Cancer statistics, 2014, CA: A Cancer Journal for Clinicians 2014;64:9-29.

[4] A. Sendler et al., Preoperative Evaluation of Pancreatic Masses with Positron Emission Tomography Using 18 F-fluorodeoxyglucose: Diagnostic Limitations, World J. Surg. 24, 1121-1129, 2000

[5] H. Jadvar, Imaging evaluation of prostate cancer with 18 F-fluorodeoxyglucose PET/CT: utility and limitations, Eur J Nucl Med Mol Imaging (2013) 40 (Suppl 1):S5S10 
[6] E. Garutti et al., EndoTOFPET-US a novel multimodal tool for endoscopy and Positron Emission Tomography, IEEE NSS/MIC Conf. Record (2012) 2096-2101. http://ieeexplore.ieee.org/ xpl/articleDetails . jsp?arnumber $=6551231$

[7] E. Roncali et al., Application of Silicon Photomultipliers to Positron Emission Tomography, Annals of Biomedical Engineering, (2011) Vol. 39, No. 4, pp. 1358-1377.

[8] P. Buzhan et al., An advanced study of silicon photomultiplier, ICFA Instrum Bull. (2001) 23:28-42.

[9] M.D Rolo et al., TOFPET ASIC for PET applications, JINST (2013) 8 C02050.

[10] Wei Shen et al., STiC - A mixed Mode Chip for SiPM ToF Applications, IEEE NSS/MIC Conf. Record (2012) N14-37.

[11] A. Tadday et al., Characterisation Studies of Silicon Photomultipliers, Nucl. Instrum. Meth. Phys. Res. A, (2010) 620217.
[12] G. Lutz, Semiconductor radiation detectors: device physics, Springer, (1999).

[13] R. Pagano et al., Dark Current in Silicon Photomultiplier Pixels: Data and Model, IEEE transactions on electron devices, vol. 59, no. 9, pp. 2410-2416, Sept. 2012.

[14] J. Trummer et al., Scintillation Properties of LuYAP and LYSO Crystals Measured with MiniACCOS, an Automatic Crystal Quality Control System, IEEE NSS/MIC Conf. Record (2005) 2807-2810.

[15] Y. Shao, A new timing model for calculating the intrinsic timing resolution of a scintillator detector, Phys. Med. Biol. 52 (2007) 1103-1117.

[16] F. Anghinolfi et al., NINO: An ultra-fast and lowpower front-end amplifier/discriminator ASIC designed for the multigap resistive plate chamber, Nucl. Instrum. Meth. A 533 (2004) 183.

[17] J.Christiansen, HPTDC, High Performance Time to Digital Converter version 2.2, CERN-EP/MIC (2004), 